\title{
ApoE and apoC-III-defined HDL subtypes: a descriptive study of their lecithin cholesterol acyl transferase and cholesteryl ester transfer protein content and activity
}

Mateo Amaya-Montoya ${ }^{1}$, Jairo A. Pinzón-Cortés', Lina S. Silva-Bermúdez', Daniel Ruiz-Manco', Maria C. Pérez-Matos ${ }^{1}$, Mario A. Jiménez-Mora ${ }^{1}$ and Carlos O. Mendivil ${ }^{1,2^{*}}$ (D)

\begin{abstract}
Background: The functionality of high-density lipoproteins (HDL) is a better cardiovascular risk predictor than HDL concentrations. One of the key elements of HDL functionality is its apolipoprotein composition. Lecithin-cholesterol acyl transferase (LCAT) and cholesterol-ester transfer protein (CETP) are enzymes involved in HDL-mediated reverse cholesterol transport. This study assessed the concentration and activity of LCAT and CETP in HDL subspecies defined by their content of apolipoproteins E (apoE) and C-III (apoC-III) in humans.

Methods: Eighteen adults (ten women and eight men, mean age 55.6, BMl $26.9 \mathrm{Kg} / \mathrm{m}^{2}, \mathrm{HbA} 1 \mathrm{c} 5.4 \%$ ) were studied. HDL from each participant were isolated and divided into four subspecies containing respectively: No apoE and no apoC-III (E-C-), apoE but not apoC-III (E+C-), apoC-III but no apoE (E-C+) and both apoE and apoC-III $(E+C+)$. The concentration and enzymatic activity of LCAT and CETP were measured within each HDL subspecies using immunoenzymatic and fluorometric methods. Additionally, the size distribution of HDL in each apolipoprotein-defined fraction was determined using nondenaturing electrophoresis and anti-apoA-I western blotting.

Results: HDL without apoE or apoC-III was the predominant HDL subtype. The size distribution of HDL was very similar in all the four apolipoprotein-defined subtypes. LCAT was most abundant in E-C- HDL (3.58 mg/mL, 59.6\% of plasma LCAT mass), while HDL with apoE or apoC-III had much less LCAT $(19.8,12.2$ and $8.37 \%$ of plasma LCAT respectively for $E+C-$, E-C + and $E+C+$ ). $L C A T$ mass was lower in $E+C-H D L$ relative to $E-C-H D L$, but LCAT activity was similar in both fractions, signaling a greater activity-to-mass ratio associated with the presence of apoE. Both CETP mass and CETP activity showed only slight variations across HDL subspecies. There was an inverse correlation between plasma LCAT activity and concentrations of both $\mathrm{E}-\mathrm{C}+$ pre-beta HDL $(r=-0.55, P=0.017)$ and $\mathrm{E}-\mathrm{C}$ - alpha $1 \mathrm{HDL}(r=-0.49, P=0.041)$. Conversely, there was a direct correlation between plasma CETP activity and concentrations of E-C+ alpha $1 \mathrm{HDL}(r=0.52, P=0.025)$.

(Continued on next page)
\end{abstract}

\footnotetext{
* Correspondence: cmendivi@uniandes.edu.co; carlosolimpo@gmail.com

'Universidad de los Andes Medical School, Carrera 7 \# 116-05, Of. 413,

Bogotá, Colombia

2Department of Internal Medicine, Fundación Santa Fe de Bogotá, Section of

Endocrinology, Carrera 7 No. 117 - 15, Bogotá, Colombia
}

(c) The Author(s). 2020 Open Access This article is licensed under a Creative Commons Attribution 4.0 International License, which permits use, sharing, adaptation, distribution and reproduction in any medium or format, as long as you give appropriate credit to the original author(s) and the source, provide a link to the Creative Commons licence, and indicate if changes were made. The images or other third party material in this article are included in the article's Creative Commons licence, unless indicated otherwise in a credit line to the material. If material is not included in the article's Creative Commons licence and your intended use is not permitted by statutory regulation or exceeds the permitted use, you will need to obtain permission directly from the copyright holder. To view a copy of this licence, visit http://creativecommons.org/licenses/by/4.0/ The Creative Commons Public Domain Dedication waiver (http://creativecommons.org/publicdomain/zero/1.0/) applies to the data made available in this article, unless otherwise stated in a credit line to the data. 
(Continued from previous page)

Conclusions: The presence of apoE in small HDL is correlated with increased LCAT activity and esterification of plasma cholesterol. These results favor an interpretation that LCAT and apoE interact to enhance anti-atherogenic pathways of HDL.

Keywords: HDL, Lecithin cholesterol acyltransferase, Cholesterol ester transfer protein, Apolipoprotein E, Apolipoprotein C-III, Reverse cholesterol transport

\section{Background}

Evidence from multiple observational studies has demonstrated a negative association between plasma concentrations of high-density lipoprotein cholesterol (HDL-C) and the risk of cardiovascular disease (CVD) [1-4]. However, medications aimed at raising HDL-C have failed to reduce the incidence of CVD in clinical trials. Several highdensity lipoprotein (HDL)-raising agents have failed to prevent CVD including cholesteryl ester transfer protein (CETP) inhibitors, fibrates and niacin [5-8]. This apparent paradox can be explained by the fact that HDL functionality, rather than HDL-C concentration, is the relevant measure associated with CVD prevention $[9,10]$. HDL functionality is a broad concept that includes reverse cholesterol transport (RCT), induction of nitric oxide synthesis, reduction in the expression of cell adhesion molecules and antioxidant activity [11]. Lecithin-cholesterol acyl transferase (LCAT) and CETP are enzymes strongly involved in HDL metabolism and functionality. LCAT transfers an acyl group from lecithin to free cholesterol, forming cholesterol esters that move to the core of the HDL particle and are later taken up by the liver $[12,13]$. Meanwhile, CETP catalyzes the transfer of cholesterol esters from HDL to apoB lipoproteins in exchange for triglycerides [14]. Subsequently, these apoB lipoproteins are also removed from circulation by the liver, completing an indirect pathway of RCT [15].

The concentration and activity of LCAT and CETP are modulated by different factors. For example, polyunsaturated fatty acids (PUFA) reduce expression of the LCAT gene and synthesis of LCAT in vitro [16]. Sphingomyelin [17], oxidized lipids [18, 19], $n-3$ fatty acids [20] and trans-unsaturated fatty acids [21] inhibit LCAT in vitro. Meanwhile, a high dietary intake of cholesterol or omega3 PUFA and the use of fibrates upregulate expression of the CETP gene [22, 23]. Plasma CETP activity and mass are also increased in parallel with higher plasma concentrations of bile acids [24], and decreased in patients with hypothyroidism [25]. Nonetheless, the modulation of human LCAT and CETP by components of HDL in vivo is insufficiently understood.

All lipoproteins harbor a repertoire of small apolipoproteins, which act as modulators of their metabolic fate. Two of these small apolipoproteins are apoE and apoCIII. ApoE mediates the clearance of very low-density lipoproteins (VLDL), intermediate-density lipoproteins (IDL) and chylomicron remnants via the low-density lipoprotein (LDL) receptor, LDL receptor-related protein-1 (LRP-1) or heparan sulfate proteoglycans [26, 27]. Contrastingly, apoC-III strongly inhibits the clearance of all apoBlipoproteins $[28,29]$. ApoC-III also impairs the catabolism of triglyceride-rich lipoproteins and stimulates hepatic VLDL assembly and secretion [28, 30]. However, the role of apoE and apoC-III on HDL physiology is much less understood. It is known that besides apoA-I (the natural cofactor of LCAT) [31], apoE is able to activate LCAT in vitro [32], while an increase in the apoC-III content of synthetic HDL exerts the opposite effect [33]. ApoE is able to partially rescue LCAT activity In apoA-I knockout mice, albeit only in VLDL and LDL, not in HDL [34]. Despite their major relevance in lipoprotein metabolism, the influence of apoE and apoC-III over LCAT and CETP in humans is very poorly understood.

With this background, this study analyzed the distribution of LCAT and CETP mass and activity in multiple HDL subclasses defined by their content of apoE and apoC-III and molecular size, in normal weight and overweight adult humans.

\section{Methods}

\section{Study design and participants}

The study included 18 participants between 33 and 76 years old, selected from a project about new biomarkers of insulin resistance [34]. Exclusion criteria were known diabetes mellitus or use of anti-diabetic medications, other endocrine disorders, diseases of the exocrine pancreas, pregnancy or use of oral anticoagulants or lipidlowering drugs. Individuals who had symptoms of an acute viral or bacterial infection, or with plasma highsensitivity C-reactive protein (hSCRP) concentrations higher than $10 \mathrm{mg} / \mathrm{L}$, were also excluded. Blood samples were drawn in EDTA (ethylenediaminetetraacetic acid) tubes after an 8-h fast. Plasma was promptly separated, supplemented with a preserving cocktail (benzamidine, phenylmethylsulphonyl fluoride and gentamicin), aliquoted and stored at $-80^{\circ} \mathrm{C}$ for later analyses. Plasma concentrations of apoA-I were measured using a nephelometric method [35] and fasting plasma glucose (FPG), plasma lipids and creatinine using conventional colorimetric assays (Biosystems, Barcelona, Spain). Glycated hemoglobin A1c (HbA1c) was determined using a National Glycohemoglobin Standardization Program- 
certified boronate affinity technique (NycoCard ${ }^{\mathrm{Tm}}$ Reader II, Alere Technologies, Oslo, Norway).

\section{$\mathrm{HDL}$ isolation and separation}

Plasma was passed through Acrodisc ${ }^{\circ} 5 \mu \mathrm{m}$ filters (Pall Corporation, Port Washington, NY, USA), in order to remove fibrin impurities. Then, HDL was purified from plasma using immunoaffinity chromatography as follows: Sepharose $4 \mathrm{~B}^{\mathrm{m}}$ resin bound to goat polyclonal antihuman apoA-I antibodies (Academy Bio-Medical, Houston, Texas, USA) was loaded into $10 \mathrm{~mL}$ Poly-Prep ${ }^{\circ}$ chromatography columns (Bio-Rad, Hercules, California, USA), and $1 \mathrm{~mL}$ of plasma was incubated overnight in the column. The unbound fraction was collected by gravity flow and stored at $-80^{\circ} \mathrm{C}$. The bound fraction was eluted using 3 consecutive washes of $3 \mathrm{M}$ sodium thiocyanate $(\mathrm{NaSCN})$ and one last phosphate-buffered saline (PBS) wash followed by concentration and desalting in $10 \mathrm{KDa}$ molecular weight cutoff Amicon ${ }^{\circ}$ filters (Merck Millipore, Billerica, MA, USA) until $1 \mathrm{~mL}$ of fraction was achieved. Then two PBS washes were performed to remove any $\mathrm{NaSCN}$ left over in the column before another use. The same process was carried out with columns containing goat polyclonal anti-human apoC-III and anti-human apo-E antibodies, in that order. At the end, total HDL from each participant was divided in 4 subfractions: HDL without apoE or apoC-III (E-C-), HDL with apoE but without apoC-III $(E+C-)$, HDL without apoE but with apoC-III (E-C+) and HDL with both apoE and apoC-III $(\mathrm{E}+\mathrm{C}+)$. The efficiency of the immunoaffinity columns was $95-98 \%$ for all study subjects. The final buffer for all HDL subfractions was PBS.

\section{Determination of enzymatic content and activity in HDL subfractions}

LCAT concentration was determined using an immunoenzymatic, double-sandwich assay (ALPCO Diagnostics, Salem, NH, USA), in which capture is performed by a first monoclonal antibody against LCAT (MoAb 36, 486) and detection is performed with a different, horseradish peroxidase (HRP)-labeled anti-LCAT monoclonal antibody (MoAb 36,487). After incubation with a substrate solution and termination with a stop reagent, the intensity of absorbance at $492 \mathrm{~nm}$ was read in a Synergy HT microplate reader using Gen5 software (BioTek, Winooski, VT, USA). CETP concentration was determined using an immunoenzymatic sandwich assay (ALPCO Diagnostics, Salem, NH, USA), with MoAb 3-11D as capture antibody and HRP-labeled MoAb 14-8F as detection antibody.

Measurement of LCAT activity was done using a fluorometric assay (Calbiochem, Darmstadt, Germany). The assay is based on the incubation of a substrate that fluoresces at $470 \mathrm{~nm}$ with the study samples. After
LCAT in the samples removes a fatty acid from the substrate and transfers it to cholesterol, the substrate loses fluorescence at $470 \mathrm{~nm}$ and gains fluorescence at 390 $\mathrm{nm}$. Thus, LCAT activity was measured as change in 470/390 emission intensity by comparison against a calibration curve based on dilutions of a plasma pool. CETP was also measured with a fluorometric assay (Abcam, Cambridge, UK). The assay principle involves incubating the sample with a mixture that contains a self-quenched fluorescent neutral lipid and an acceptor molecule. Action of CETP in the sample results in transfer of the neutral lipid to the acceptor molecule and an increase in its fluorescence (excitation at $465 \mathrm{~nm}$, emission at 535 $\mathrm{nm})$. For CETP too, activity was measured by comparison against a calibration curve based on dilutions of a plasma pool. Enzymatic activity was expressed as percent of activity in the plasma pool, and was therefore expressed in Arbitrary Units (AU). All measurements were performed in duplicate.

The cholesteryl ester content of plasma and HDL subfractions was measured using the Abcam cholesteryl ester kit (ab65359, Abcam, Cambridge, UK). The assay is based on two simultaneous reactions for cholesterol determination, one of which includes cholesterol esterase as part of the reaction mixture, while the other does not. Cholesteryl esters are then calculated by subtraction as (total cholesterol - free cholesterol). Phosphatidylcholine in plasma and HDL subfractions was measured using the Abcam fluorometric assay (ab83377, Abcam, Cambridge, UK). The test is based on an enzyme-coupled reaction that hydrolyzes phosphatidylcholine and releases choline, which in turn reacts with the OxiRed probe and generates fluorescence at $587 \mathrm{~nm}$ wavelength.

\section{Determination of HDL size distribution}

HDL contained in each of the four apoC-III and apoE-defined fractions were separated by size using non-denaturing polyacrylamide gradient gel electrophoresis (NDPAGGE). Twenty-five microliters of each immunofraction plus $25 \mu \mathrm{l}$ of sample buffer were loaded into a 4-30\% polyacrylamide gradient gel (Jule Inc., Milford, CT, USA). Wells 1 and 10 were loaded with molecular size standards (Amersham HMW Native Marker Kit, GE Healthcare, Little Chalfont, UK) and gels were run for $24 \mathrm{~h}$ at constant $70 \mathrm{~V}$. Then, contents of the gel were transferred to a $0.45 \mu \mathrm{m}$ pore size polyvinylidene fluoride (PVDF) membrane (Pall Corporation, New York, NY, USA) in a wet transfer apparatus at $30 \mathrm{~V}$ for $24 \mathrm{~h}$. The lanes containing the MW markers were cut from the rest of the membrane, stained in $0.2 \%$ amido black solution for 20 min and stored for later photographing. The rest of the membrane was blocked with $5 \%$ powder low-fat 
milk, incubated with an HRP-conjugated goat antihuman apoA-I antibody (Academy Bio-medical, Houston, TX, USA), and revealed using 3,3',5,5'-tetramethylbenzidine as substrate. Later, the marker lanes and the rest of the membrane were placed side to side and photographed in a Bio-Rad ChemiDoc ${ }^{\mathrm{Tm}}$ MP gel documenter. Using the molecular size standards as reference, the intensity of the bands/smears in each size range fractions was quantitated in Image $\mathrm{Lab}^{\mathrm{Tx}}$ software. Size fractions were defined as follows: prebeta HDL: < $7.1 \mathrm{~nm}$, alpha 3 HDL: $7.1-8.2 \mathrm{~nm}$, alpha 2 HDL: $8.2-9.5 \mathrm{~nm}$ and alpha 1 HDL: $9.5-12.2 \mathrm{~nm}$. The concentration of apoA-I in each HDL size subfraction was estimated by multiplying the proportion of apoA-I within that fraction by the directly measured total plasma apoA-I. Laboratory procedures were executed at the Diabetes, Lipids and Metabolism laboratory of Universidad de Los Andes, following current institutional biosafety protocols.

\section{Statistical analyses}

A sample size of eighteen participants provided $87 \%$ power to detect a true difference of at least $8 \%$ in LCAT activity between two HDL subtypes, assuming a $5 \%$ variation coefficient in LCAT activity [18], at a 5\% significance level. The distribution of plasma apoA-I across HDL subfractions was compared in a 2-way analysis of variance (ANOVA) model in which apoA-I concentration in each subfraction was the dependent variable, and HDL size and immunofraction were fixed factors. Enzyme concentrations and activities were compared among HDL subtypes using a 1-way ANOVA in which HDL immunofraction was the only fixed factor. When global ANOVA was significant, post-hoc comparisons against the reference fraction (E-C-) were done using Scheffé's method. Comparisons of numeric variables between groups of participants were performed using MannWhitney's U test. The significance of differences in proportions between groups of participants was assessed using Fisher's exact test. All tests were done at a 0.05 significance level and all reported $p$-values are 2 -sided.

\section{Results}

The study included eight male and ten female participants, with mean age $55.6+/-11.2$ years and mean body-mass index (BMI) $26.9+/-4.0 \mathrm{~kg} / \mathrm{m}^{2}$. On average, women had higher plasma total cholesterol, HDLc and LDLc. Women also had significantly higher plasma apoA-I levels $(121.4+/-14.3 \mathrm{mg} / \mathrm{dL}$ versus $97.7+/-$ $23.0 \mathrm{mg} / \mathrm{dL}$ in men, $P=0.016$ ). Plasma LCAT concentration was $6.3+/-2.2 \mathrm{mg} / \mathrm{dL}$ in women and $5.7+/-2.2$ $\mathrm{mg} / \mathrm{dL}$ in men, while plasma CETP concentrations were $1.9+/-0.7 \mathrm{mg} / \mathrm{dL}$ in women and $1.7+/-0.7 \mathrm{mg} / \mathrm{dL}$ in men. The relative activity of the enzymes under study was not different between sexes: $98.7+/-13.7 \mathrm{AU}$ in
Table 1 Characteristics of study participants. eGFR: Estimated glomerular filtration rate, CETP: Cholesteryl ester transfer protein, LCAT: Lecithin cholesterol acyl transferase. Data are mean +/SD unless stated otherwise

\begin{tabular}{ll}
\hline Sex (F:M) & $10: 8$ \\
Age (years) & $55.6+/-11.2$ \\
Weight (kg) & $72.0+/-12.9$ \\
Height (m) & $163.5+/-9.2$ \\
Body-mass index (kg/m²) & $26.9+/-4.0$ \\
Percent body fat (\%) & $33.1+/-7.1$ \\
Percent abdominal fat (\%) & $9.4+/-3.9$ \\
Percent lean mass (\%) & $63.6+/-6.7$ \\
Systolic blood pressure (mmHg) & $117.8+/-14.6$ \\
Diastolic blood pressure (mmHg) & $73.8+/-11$ \\
Fasting plasma glucose (mg/dl) & $96.1+/-10.6$ \\
Glycated hemoglobin (HbA1c) (\%) & $5.4+/-1.0$ \\
eGFR (ml/min) & $93.2+/-18.0$ \\
Total cholesterol (mg/dl) & $195.5+/-52.4$ \\
Triglycerides (mg/dl) & $154.2+/-58.4$ \\
HDL cholesterol (mg/dl) & $43.8+/-15.8$ \\
LDL cholesterol (mg/dl) & $126.3+/-44.4$ \\
C-reactive protein (mg/l) & $1.9+/-2.6$ \\
Plasma apoA-l (mg/dl) & $110.9+/-21.8$ \\
Plasma CETP (microg/ml) & $1.8+/-0.7$ \\
Plasma LCAT (microg/ml) & $6.0+/-2.1$ \\
Plasma CETP activity (AU) & $100.0+/-10.8$ \\
Plasma LCAT activity (AU) & $96.4+/-20.1$ \\
\hline &
\end{tabular}

women versus $101.7+/-6.1 \mathrm{AU}$ in men for CETP and $96.1+/-22.3 \mathrm{AU}$ in women versus $96.8+/-18.4 \mathrm{AU}$ in men for LCAT (Table 1).

\section{HDL with apoE or apoC-III are minority}

The predominant subfraction of HDL (as reflected by its concentration of apoA-I) was E-C- in most study participants. On average, E-C- HDL represented $50.1 \%$ of plasma apoA-I, followed by $\mathrm{E}+\mathrm{C}-(22.6 \%), \mathrm{E}-\mathrm{C}+(15.2 \%)$ and $\mathrm{E}+\mathrm{C}+(12.1 \%)(p<0.001$ for difference across subfractions) (Fig. 1). There was significantly more apoA-I in $\mathrm{E}-\mathrm{C}$ - than in $\mathrm{E}+\mathrm{C}-\mathrm{E}-\mathrm{E}+$ or $\mathrm{E}+\mathrm{C}+\mathrm{HDL}(p<0.001$ for each of the three pairwise comparisons). Overall, $34.7 \%$ of HDL contained any apoE while $27.3 \%$ contained any apoC-III.

\section{LCAT concentration and activity vary across HDL subfractions}

Most plasma LCAT mass (3.58 microg/mL, 59.6\%) was concentrated in the E-C- HDL subfraction. The remaining 


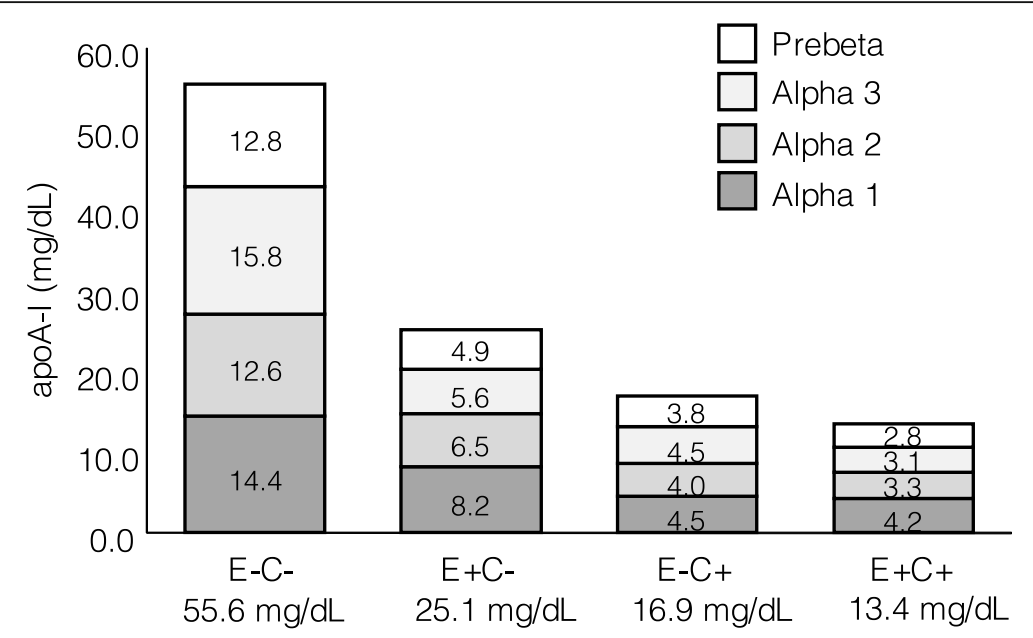

Fig. 1 Distribution of plasma apoA-I across HDL subfractions defined by size, apoE and apoC-III content. HDL size ranges are as follows: Prebeta: $<7.1 \mathrm{~nm}$, alpha 3: 7.1-8.2 nm, alpha 2: 8.2-9.5 nm, alpha 1: 9.5-12.2 $\mathrm{nm}(n=18,10$ women and 8 men)
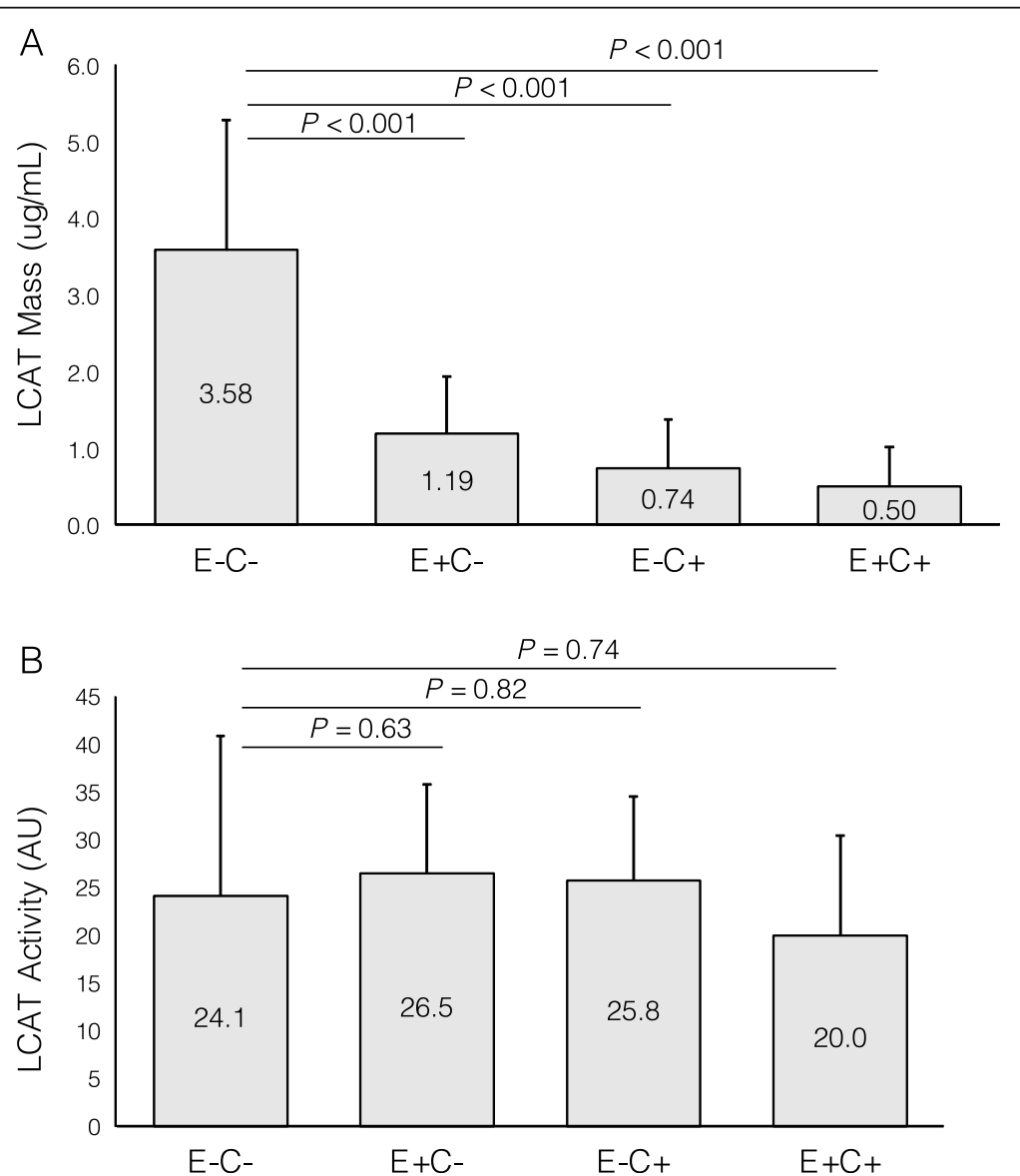

Fig. 2 Lecithin cholesterol acyl transferase (LCAT) concentration and activity in HDL subfractions defined by their apoE and apoC-III content. a LCAT concentration. b LCAT activity ( $n=18,10$ women and 8 men) 
fractions with either apoE or apoC-III had similarly lower LCAT concentrations: $1.19 \mathrm{microg} / \mathrm{mL}$ in $\mathrm{E}+\mathrm{C}-, \quad 0.74$ microg $/ \mathrm{mL}$ in $\mathrm{E}-\mathrm{C}+$ and $0.50 \mathrm{microg} / \mathrm{mL}$ in $\mathrm{E}+\mathrm{C}+(p<$ 0.001 for the difference across groups). The difference in LCAT concentration between E-C- and every other HDL subfraction was statistically significant $(p<0.001$ for each of the three pairwise comparisons) (Fig. 2, upper panel). Surprisingly, this heterogeneity in LCAT protein distribution was not paralleled by LCAT activity, which had a different distribution. The E-C- fraction had a relative LCAT activity of $24.1 \mathrm{AU}$ ( $25 \%$ of total plasma LCAT activity), and the highest LCAT activity was found in the in $\mathrm{E}+\mathrm{C}$ - fraction (26.5 AU, 27.4\% of total plasma LCAT activity) ( $P=0.35$ for difference across groups). HDL in the E-C+ and $\mathrm{E}+\mathrm{C}+$ fractions contained respectively 25.8 and 20.0 AU of LCAT activity (Fig. 2, lower panel). The ratio of LCAT activity / LCAT mass was $27.5 \mathrm{AU}$ "mL/microg in $\mathrm{HDL}$ containing apoE (E + $\mathrm{C}$ - plus $\mathrm{E}+\mathrm{C}+$ ), and 11.6 $\mathrm{AU}$ * $\mathrm{mL} /$ microg in $\mathrm{HDL}$ not containing apoE (E-C-plus E-C+).
CETP concentration and activity are similar across HDL subfractions

Most CETP was concentrated in the $\mathrm{E}+\mathrm{C}$ - subfraction $(0.60 \mathrm{mg} / \mathrm{mL}, 32.5 \%$ of total plasma CETP), while the other three types of HDL contained respectively 25.9\% (EC-), 21.1\% (E-C+) and 20.4\% (E + C+) of plasma CETP $(P=0.49$ for comparison across fractions) (Fig. 3, upper panel). CETP activity showed a very homogeneous distribution across HDL subtypes ( $P=0.50$, Fig. 3 , lower panel).

\section{Lipid composition of HDL subtypes}

ApoC-III containing HDL displayed a higher triglyceride-to-apoA1 molar ratio, while E-C- HDL were poorer in triglycerides relative to the other fractions $(P=0.01$ for global ANOVA, Table 2). Despite numerically higher phosphatidylcholine-to-apoA1 molar ratios in the apoE or apoC-III containing HDL, this difference did not achieve statistical significance. The cholesterol and cholesteryl ester contents of the
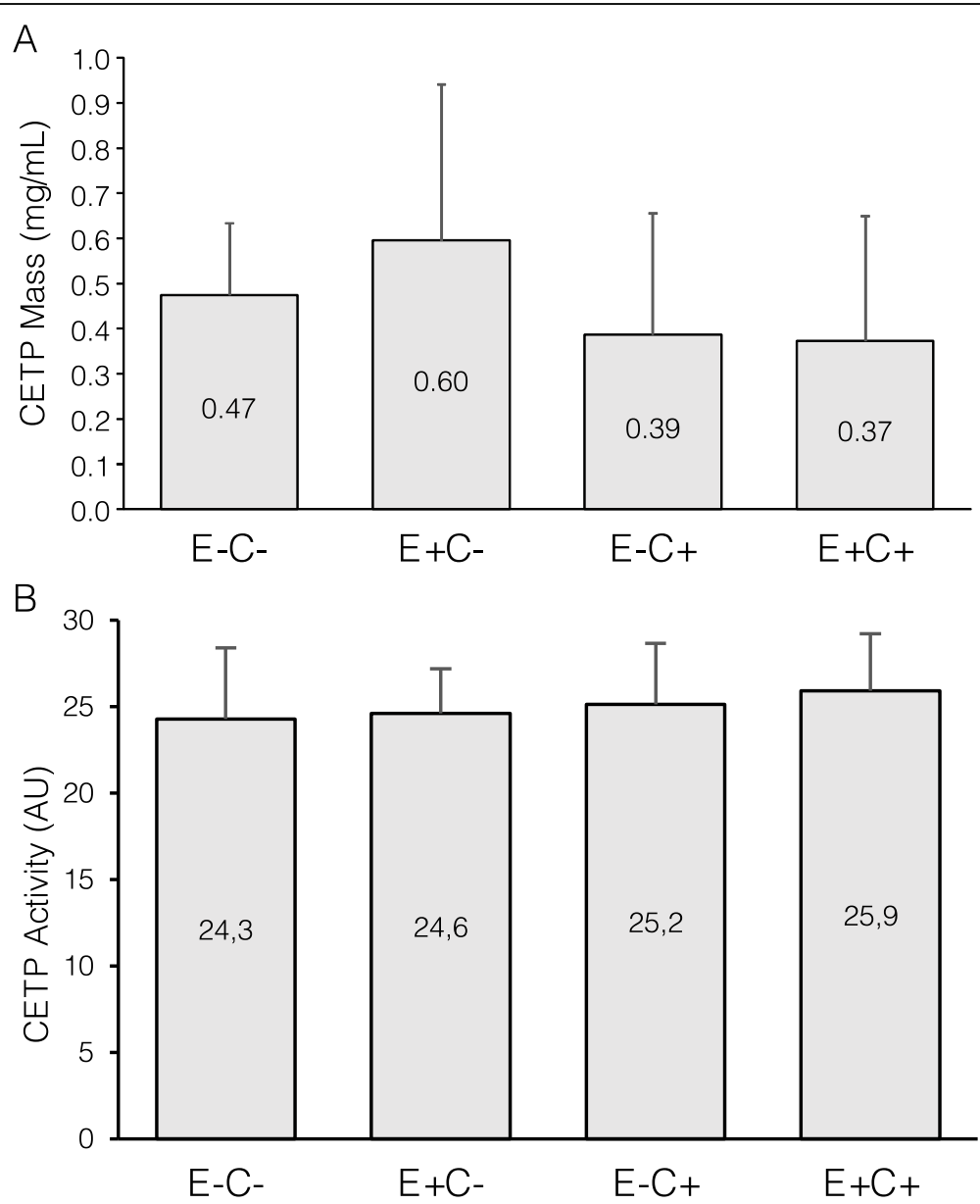

Fig. 3 Cholesteryl ester transfer protein (CETP) concentration and activity in HDL subfractions defined by their apoE and apoC-III content. a CETP concentration. There was no significant difference across subfractions (overall ANOVA $P=0.48$ ). b CETP activity. There was no significant difference across subfractions (overall ANOVA $P=0.50)(n=18,10$ women and 8 men) 
Table 2 Molar ratios of lipids to apoA-I in the four HDL subfractions. Data in the first row are mean lipid to apoA-I molar ratios +/$\mathrm{SD}$. The second row of each analyte shows the significance values from a post-hoc pairwise comparison versus the E-CIII- fraction. The third row of each analyte shows the proportion of each lipid in that fraction as a percentage of the total mass of such lipid in HDL. The last row shows the significance value from a global one-way ANOVA for the analysis of lipid to apoA-I molar ratios by subfractions

\begin{tabular}{|c|c|c|c|c|c|}
\hline & & E-CIII- & $E+C I I I-$ & $\mathrm{E}-\mathrm{Clll}+$ & $\mathrm{E}+\mathrm{CIII+}$ \\
\hline \multirow[t]{4}{*}{ Triglycerides } & to apoA-I molar ratio & $0.47+/-0.22$ & $1.09+/-1.41$ & $2.73+/-3.48$ & $2.41+/-1.87$ \\
\hline & $p$-value versus E-CIII- & - & 0.88 & 0.035 & 0.09 \\
\hline & $\%$ of total mass in HDL & $25.2 \%$ & $17.2 \%$ & $29.8 \%$ & $27.7 \%$ \\
\hline & $p$-value from ANOVA & 0.01 & & & \\
\hline \multirow[t]{4}{*}{ Cholesterol } & to apoA-I molar ratio & $26.6+/-18.7$ & $51.5+/-68.4$ & $48.1+/-32$ & $52.6+/-38.1$ \\
\hline & $p$-value versus E-CIII- & - & 0.43 & 0.55 & 0.39 \\
\hline & $\%$ of total mass in $\mathrm{HDL}$ & $40.7 \%$ & $22.5 \%$ & $20.8 \%$ & $15.8 \%$ \\
\hline & $p$-value from ANOVA & 0.26 & & & \\
\hline \multirow[t]{4}{*}{ Phosphatidylcholine } & to apoA-I molar ratio & $109.2+/-69.3$ & $317.6+/-367.7$ & $455.7+/-547.3$ & $488.2+/-669.2$ \\
\hline & $p$-value versus E-CIII- & - & 0.65 & 0.21 & 0.15 \\
\hline & $\%$ of total mass in $\mathrm{HDL}$ & $27.1 \%$ & $27.0 \%$ & $26.0 \%$ & $19.7 \%$ \\
\hline & $p$-value from ANOVA & 0.088 & & & \\
\hline \multirow[t]{4}{*}{ Cholesterol esters } & to apoA-I molar ratio & $14.6+/-16$ & $23+/-29.9$ & $16.3+/-37.9$ & $30+/-103$ \\
\hline & $p$-value versus E-CIII- & - & 0.98 & 0.99 & 0.89 \\
\hline & $\%$ of total mass in $\mathrm{HDL}$ & $48.6 \%$ & $28.8 \%$ & $12.3 \%$ & $10.1 \%$ \\
\hline & $p$-value from ANOVA & 0.86 & & & \\
\hline
\end{tabular}

four HDL subtypes were numerically similar, and not statistically different.

\section{The concentration of some apolipoprotein and size- defined HDL correlates with plasma LCAT and CETP activity}

The concentration of apoA-I in the E-C+ pre-beta HDL subfraction correlated inversely with total plasma LCAT activity $(r=-0.55, P=0.017$, Table 3$)$. Similarly, apoA-I concentrations in the E-C- alpha $1 \mathrm{HDL}$ subfraction correlated inversely with total plasma LCAT activity $(\mathrm{r}=-$

Table 3 Correlations between apoA-I mass within HDL sizedefined subfractions and plasma enzymatic activities. Data are Spearman linear correlation coefficients. Asterisks denote correlations significantly different from zero

\begin{tabular}{|c|c|c|c|c|}
\hline Correlations with LCAT activity & E-CIII- & $\mathrm{E}+\mathrm{CIII-}$ & $\mathrm{E}-\mathrm{CIII+}$ & $\mathrm{E}+\mathrm{CIII+}$ \\
\hline Alpha $1 \mathrm{HDL}$ & $-0.49^{*}$ & -0.30 & -0.35 & -0.10 \\
\hline Alpha $2 \mathrm{HDL}$ & -0.01 & -0.29 & -0.37 & 0.02 \\
\hline Alpha $3 \mathrm{HDL}$ & 0.33 & -0.13 & -0.30 & 0.04 \\
\hline Pre-Beta HDL & 0.24 & -0.08 & $-0.55^{*}$ & 0.06 \\
\hline Correlations with CETP activity & E-CIII- & $\mathrm{E}+\mathrm{CIII-}$ & $\mathrm{E}-\mathrm{CIII+}$ & $\mathrm{E}+\mathrm{CIII+}$ \\
\hline Alpha $1 \mathrm{HDL}$ & 0.16 & -0.10 & $0.52^{*}$ & 0.46 \\
\hline Alpha $2 \mathrm{HDL}$ & 0.04 & -0.07 & 0.39 & 0.34 \\
\hline Alpha $3 \mathrm{HDL}$ & -0.03 & 0.11 & 0.28 & 0.28 \\
\hline Pre-Beta HDL & 0.11 & -0.01 & 0.13 & 0.05 \\
\hline
\end{tabular}

$0.49, P=0.041)$. On the other hand, concentrations of larger E-C+ HDL (alpha 1) exhibited a positive correlation with plasma CETP activity $(r=0.52, P=0.025)$. For the E$\mathrm{C}+\mathrm{HDL}$ subtype, the correlation between HDL concentration and CETP activity was not significant for prebeta HDL, but tended to increase for HDL of larger sizes.

\section{The distribution of LCAT and CETP concentration and activity was not influenced by BMI}

As a sensitivity analysis, the findings concerning enzymatic concentrations and activities were compared between participants with a normal body-mass index $(\mathrm{BMI}<25)$, and participants with excess body weight (overweight or obesity, $\mathrm{BMI}>=25$ ). Just like in the complete study sample, in each of the two subgroups the concentration of LCAT was significantly higher in E-CHDL, but enzymatic activity was similarly distributed across the four HDL subtypes (Fig. 4). Also in each of the two BMI-defined subgroups, CETP concentration and activity were very similar among the four HDL subtypes (Fig. 5).

\section{Discussion}

The results of this in vivo study reveal the association between small apolipoproteins and the activity of enzymes involved in HDL-mediated reverse cholesterol transport. HDL with apoE have a greater ratio of LCAT activity to mass, signaling a greater degree of LCAT 


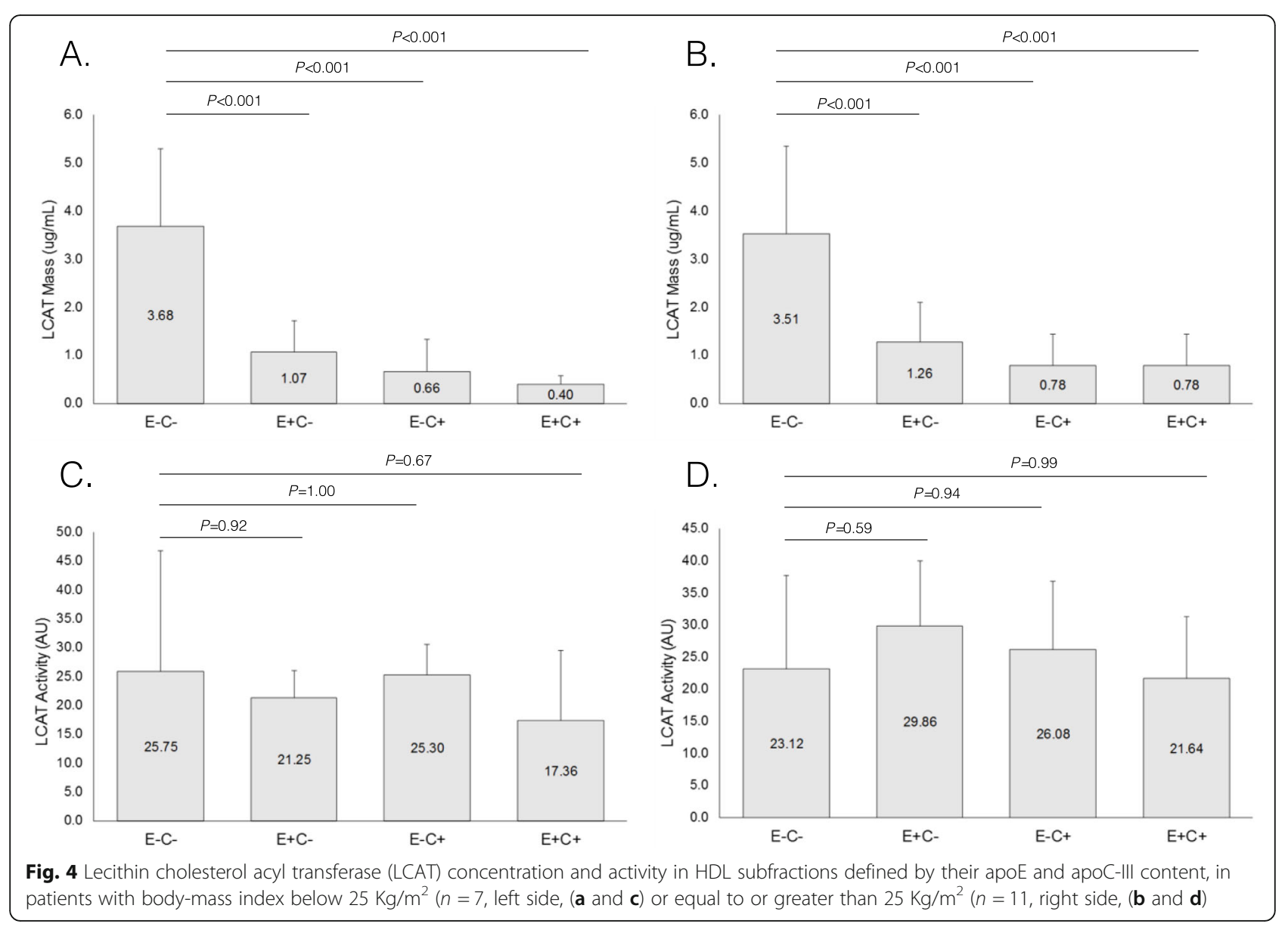

activation. Contrastingly, CETP mass and activity were not associated with the apoE or apoC-III composition of HDL. As reported previously, apoE and/or apoC-IIIcontaining HDL were a minority of total plasma HDL [36], yet they are different from E-C- HDL in their enzymatic activity. These findings suggest an interaction between LCAT and apoE in HDL that favors cholesterol esterification, a critical step for its transport to the liver.

LCAT was most abundant in E-C- HDL, while HDL with either apoE or apoC-III contained similar amounts of LCAT. Despite having lower LCAT masses compared to E-C-, HDL with apoE or apoC-III had very similar LCAT activities, suggesting that the presence of either small apolipoprotein, or a factor closely associated with them, influences LCAT. Of note, the simultaneous presence of apoE and apoC-III in HDL was not accompanied by higher LCAT activity. The essential role of apoA-I as an LCAT cofactor is well documented, but very little is known about the impact of other small apolipoproteins on the activity of this crucial enzyme. Studies in apoA-I -/-, apoE-/- double knockout mice have identified apoE as a potential LCAT activator [37]. In an in vitro model of reconstituted HDL that lacks apoA-I, apoE activates LCAT [38]. In contrast, apoC-III dose- dependently inhibits the LCAT reaction in reconstituted HDL [33], and in synthetic phosphatidylcholine vesicles containing apoA-I [39]. Therefore, the finding of no decrease in LCAT activity in apoC-III-containing HDL was unexpected.

Contrary to LCAT, the concentration and activity of CETP did not vary among HDL subtypes. Some CETP inhibitors increase both plasma apoC-III and plasma apoE [40-42], but findings from this study suggest that CETP activity and concentration in HDL are not influenced by these apolipoproteins. CETP activity in humans is known to be influenced by high plasma cholesterol concentrations, or by cholestasis $[43,44]$.

Concerning the lipid composition of HDL subtypes, there was more phosphatidylcholine in HDL with either apoE or apoC-III. Thus, higher LCAT activity in these HDL subtypes may be a consequence of greater substrate availability. This is a plausible explanation, as the movement of apolipoproteins between lipoproteins sometimes involves phospholipid transfer protein (PLTP), in which cases there may be simultaneous transfer of the surrounding phospholipid-rich membrane [45]. There was no statistically significant difference in the cholesteryl ester content of the four HDL subtypes. 


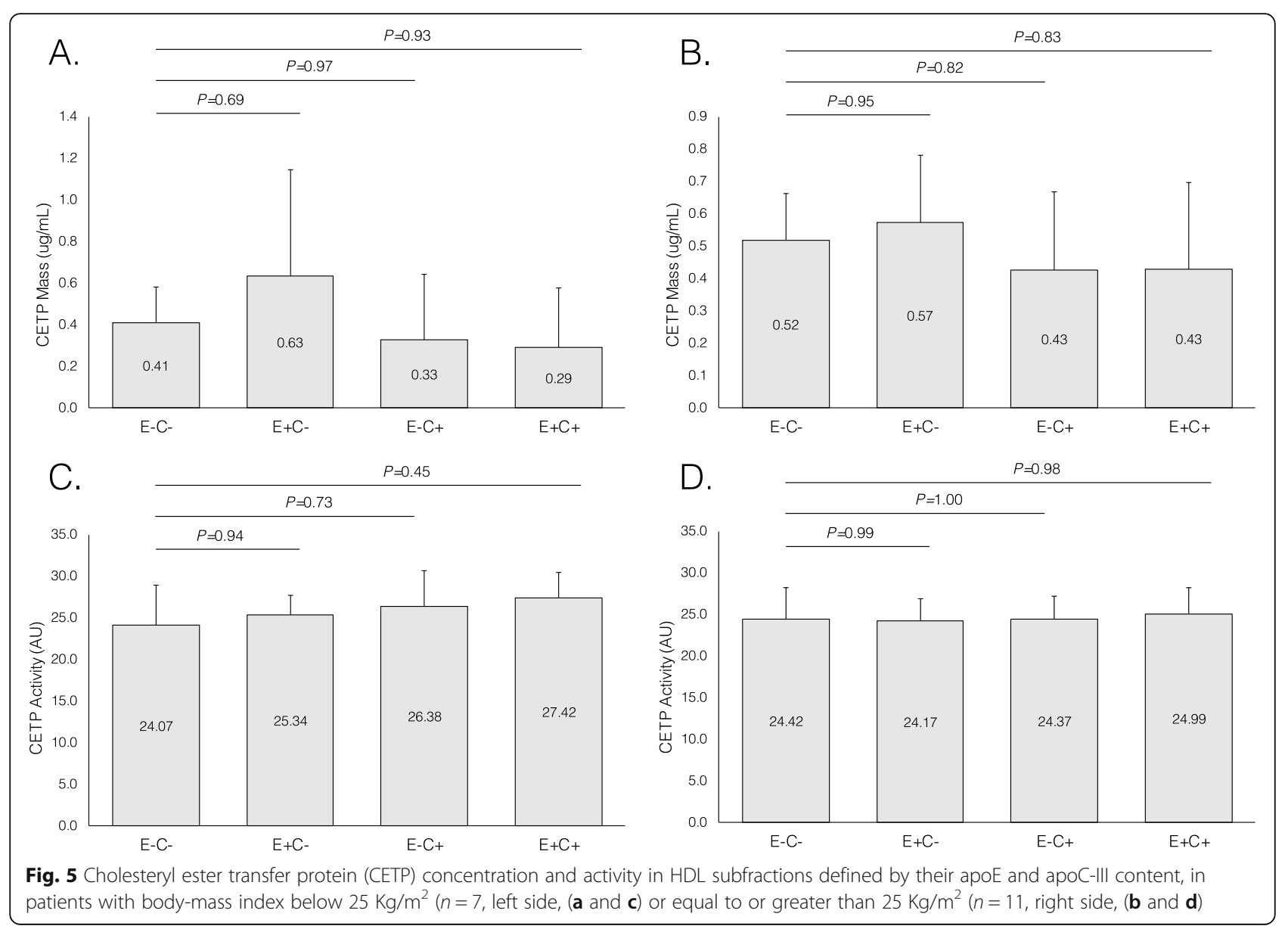

Total plasma LCAT activity showed a significant inverse correlation with the concentration of both $\mathrm{E}-\mathrm{C}+$ pre-beta HDL and E-C- alpha $1 \mathrm{HDL}$. These results suggests that the presence of apoC-III in pre-beta HDL may correlate with limited LCAT activity, less maturation and accumulation of pre-beta HDL [46]. Interestingly, a study comparing patients with and without CVD found an inverse correlation between LCAT activity and plasma pre-beta HDL concentrations that was stronger in the CVD group [47]. The negative correlation between E-C- alpha $1 \mathrm{HDL}$ and plasma LCAT activity suggests that not all size conversion/maturation of HDL requires LCAT. Meanwhile, the positive correlation of plasma CETP activity with the abundance of large E-C+ HDL may just reflect the frequent coexistence of both proteins (CETP and apoC-III) in this HDL type.

Strengths of this study include the exploration of central aspects of HDL functionality in the real physiological context of living humans. ApoE and apoC-III are biochemical antagonists [48] with an opposite impact on HDL metabolism and cardiovascular risk [49], making them particularly worthy of investigation. The finding of an association between apoE in HDL and cholesterol esterification has clinical relevance, because the influence of apoE on HDL metabolism is susceptible of positive modification through dietary intervention [50].

The main limitations of the study are its limited sample size and the fact that only two specific apolipoproteins were studied, out of the many known to be present in HDL. However, this was a proof-of-concept study in which the core analyses were performed withinindividual, so that enzymatic contents and activities were performed for different HDL subtypes belonging to the same participant. Another possible limitation is that the employed LCAT activity assay probably detects only the fatty acid removal from phosphatidylcholine and not its successful transfer to free cholesterol. Nonetheless, given the known nature of the LCAT enzymatic process, the ability of LCAT to perform this initial cleavage in vitro has been widely employed as a proxy for LCAT activity.

\section{Conclusion}

The results of this study showed specific profiles of LCAT mass and activity in HDL subtypes defined by their content of apoE and apoC-III. The presence of apoE protein in HDL was a correlate of increased LCAT 
activity, suggesting that LCAT and apoE interact to enhance anti-atherogenic pathways dependent on HDL functionality. Further studies describing the changes in HDL composition, functionality and associated enzymatic activity in the context of CVD would expand on these findings.

\section{Abbreviations}

apoC-III: apolipoprotein C-III; apoE: apolipoproteins E; AU: Arbitrary Units; BMI: Body-mass index; CETP: Cholesterol-ester transfer protein; CVD: Cardiovascular disease; HDL: High density lipoproteins; HRP: Horseradish peroxidase; LCAT: Lecithin-cholesterol acyl transferase; LRP-1: LDL receptorrelated protein-1; RCT: Reverse cholesterol transport; PUFA: Polyunsaturated fatty acids; PVDF: Polyvinylidene fluoride

\section{Acknowledgements}

We want to thank the study participants for their patience and collaboration.

\section{Authors' contributions}

MA-M, JAP-C, LSS-B and COM participated in the study conception, MA-M, JAP-C, LSS-B, DR-M, MCPM, MAJM and COM executed study experiments and data collection, MA-M, DR-M and COM participated in data analysis and manuscript writing, COM directed the project and participated in all phases of its conception, execution, and in the manuscript writing. The authors read and approved the final manuscript.

\section{Funding}

This study was funded by Vicerrectoría de Investigaciones, Universidad de los Andes, Colombia.

\section{Availability of data and materials}

The datasets used and/or analysed during the current study are available from the corresponding author on reasonable request.

\section{Ethics approval and consent to participate}

The Internal Review Board (Comité de Ética) of Universidad de los Andes approved the study according to minute 307 of 2014 . We complied with all scientific, technical and administrative norms for health research dictated by resolution 008430-1993 of the Colombian Ministry of Health and with the principles stated by the Declaration of Helsinki. All study subjects underwent an informed consent procedure and provided written informed consent.

\section{Consent for publication}

Not applicable.

\section{Competing interests}

The authors declare that they have no competing interests.

Received: 3 January 2020 Accepted: 20 May 2020

Published online: 25 May 2020

\section{References}

1. Rosenson RS, Brewer HB Jr, Barter PJ, Björkegren JLM, Chapman MJ, Gaudet D, et al. HDL and atherosclerotic cardiovascular disease: genetic insights into complex biology. Nat Rev Cardiol. 2018;15:9-19.

2. Vitali C, Khetarpal SA, Rader DJ. HDL cholesterol metabolism and the risk of CHD: new insights from human genetics. Curr Cardiol Rep. 2017;19:132.

3. Nicholls SJ, Nelson AJ. HDL and cardiovascular disease. Pathology. 2019;51: 142-7.

4. Shahid M, Sun RL, Liu Y, Bao JL, Huang CX, Liao Y, et al. Is high high-density lipoprotein cholesterol beneficial for premature coronary heart disease? A meta-analysis. Eur J Prev Cardiol. 2016;23:704-13.

5. Barter P, Caulfield M, Eriksson M, Grundy S, Kastelein J, Komajda M, et al. Effects of Torcetrapib in patients at high risk for coronary events. N Engl J Med. 2007:357:2109-22.

6. Schwartz G, Olsson A, Abt M, Ballantyne C, Barter P, Brumm J, et al. Effects of Dalcetrapib in patients with a recent acute coronary syndrome. $\mathrm{N}$ Engl J Med. 2012;367:2089-99.
7. Landray M, Haynes R, Hopewell J, Parish S, Phil D, Aung T, et al. Effects of extended-release niacin with laropiprant in high-risk patients. N Engl J Med. 2014;371:203-12.

8. Lincoff A, Nicholls S, Riesmeyer J, Barter P, Brewer H, Fox K, et al. Evacetrapib and cardiovascular outcomes in high-risk vascular disease. N Engl J Med. 2017;376:1933-42.

9. Bardagjy AS, Steinberg FM. Relationship between hdl functional characteristics and cardiovascular health and potential impact of dietary patterns: a narrative review. Nutrients. 2019;11:E1231.

10. Cuchel M, Rohatgi A, Sacks FM, Guyton JR. JCL roundtable: high-density lipoprotein function and reverse cholesterol transport. J Clin Lipidol. 2018; 12:1086-94

11. Rosenson $R$, Brewer $H$, Ansell B, Barter $P$, Chapman M, Heinecke J, et al. Dysfunctional HDL and atherosclerotic cardiovascular disease. Nat Rev Cardiol. 2016;13:48-60.

12. Dobiášová M. Atherogenic impact of lecithin-cholesterol acyltransferase and its relation to cholesterol esterification rate in HDL (FER (HDL)) and AIP [log (TG/HDL-C)] biomarkers: the butterfly effect? Physiol Res. 2017;66:193-203.

13. Mendivil CO, Furtado J, Morton AM, Wang L, Sacks FM. Novel pathways of apolipoprotein A-I metabolism in high-density lipoprotein of different sizes in humans. Arterioscler Thromb Vasc Biol. 2016:36:156-65.

14. Barter P, Rye K. Cholesteryl Ester transfer protein inhibition is not yet deadpro. Arterioscler Thromb Vasc Biol. 2016;36:439-41.

15. Maranhão RC, Freitas FR. HDL metabolism and atheroprotection: predictive value of lipid transfers. Adv Clin Chem. 2014;65:1-41.

16. Kuang YL, Paulson KE, Lichtenstein AH, Lamon-Fava S. Regulation of the expression of key genes involved in HDL metabolism by unsaturated fatty acids. Br J Nutr. 2012;108:1351-9.

17. Subbaiah PV, Jiang XC, Belikova NA, Aizezi B, Huang ZH, Reardon CA. Regulation of plasma cholesterol esterification by sphingomyelin: effect of physiological variations of plasma sphingomyelin on lecithin-cholesterol acyltransferase activity. Biochim Biophys Acta. 1821;2012:908-13.

18. Nakhjavani M, Asgharani F, Khalilzadeh O, Esteghamati A, Ghaneei A, Morteza A, et al. Oxidized low-density lipoprotein is negatively correlated with lecithin-cholesterol acyltransferase activity in type 2 diabetes mellitus. Am J Med Sci. 2011;341:92-5.

19. Bielicki JK, Forte TM. Evidence that lipid hydroperoxides inhibit plasma lecithin:cholesterol acyltransferase activity. J Lipid Res. 1999:40:948-54.

20. Abbey M, Clifton P, Kestin M, Belling B, Nestel P. Effect of fish oil on lipoproteins, lecithin: cholesterol acyltransferase, and lipid transfer protein activity in humans. Arteriosclerosis. 1990;10:85-94.

21. Subbaiah PV, Subramanian VS, Liu M. Trans unsaturated fatty acids inhibit lecithin: cholesterol acyltransferase and alter its positional specificity. J Lipid Res. 1998;39:1438-47.

22. Jiang XC, Agellon LB, Walsh A, Breslow JL, Tall A. Dietary cholesterol increases transcription of the human cholesteryl ester transfer protein gene in transgenic mice. Dependence on natural flanking sequences. J Clin Invest. 1992; $90: 1290-5$

23. Raposo HF, Patrício PR, Simões MC, Oliveira HC. Fibrates and fish oil, but not corn oil, up-regulate the expression of the cholesteryl ester transfer protein (CETP) gene. J Nutr Biochem. 2014:25:669-74.

24. Gautier T, de Haan W, Grober J, Ye D, Bahr MJ, Claudel T, et al. Farnesoid X receptor activation increases cholesteryl ester transfer protein expression in humans and transgenic mice. J Lipid Res. 2013;54:2195-205.

25. Skoczyńska A, Wojakowska A, Turczyn B, Zatońska K, Wołyniec M, Rogala N, et al. Serum lipid transfer proteins in Hypothyreotic patients are inversely correlated with thyroid-stimulating hormone (TSH) levels. Med Sci Monit. 2016;22:4661-9.

26. Huang Y, Mahley RW. Apolipoprotein E: structure and function in lipid metabolism, neurobiology, and Alzheimer's diseases. Neurobiol Dis. 2014;72:3-12.

27. Mondal T, Wang H, DeKoster GT, Baban B, Gross ML, Frieden C. ApoE: in vitro studies of a small molecule effector. Biochemistry. 2016:55:2613-21.

28. Zheng C, Khoo C, Furtado J, Sacks FM. Apolipoprotein C-III and the metabolic basis for hypertriglyceridemia and the dense low-density lipoprotein phenotype. Circulation. 2010;121:1722-34.

29. Mendivil CO, Zheng C, Furtado J, Lel J, Sacks FM. Metabolism of very-lowdensity lipoprotein and low-density lipoprotein containing apolipoprotein C-III and not other small apolipoproteins. Arterioscler Thromb Vasc Biol. 2010;30:239-45

30. Qin W, Sundaram M, Wang Y, Zhou H, Zhong S, Chang CC, et al. Missense mutation in APOC3 within the C-terminal lipid binding domain of human 
ApoC-III results in impaired assembly and secretion of triacylglycerol-rich very low density lipoproteins: evidence that ApoC-III plays a major role in the formation of lipid precursors within the microsomal lumen. J Biol Chem. 2011:286:27769-80.

31. Sorci-Thomas MG, Bhat $\mathrm{S}$, Thomas MJ. Activation of lecithin:cholesterol acyltransferase by HDL ApoA-I central helices. Clin Lipidol. 2009:4:113-24.

32. Jonas A. Lecithin cholesterol acyltransferase. Biochim Biophys Acta. 2000; 1529:245-56.

33. Cho KH. Synthesis of reconstituted high density lipoprotein (rHDL) containing apoA-I and apoC-III: the functional role of apoC-III in rHDL. Mol Cell. 2009;27:291-7.

34. Toloza FJK, Pérez-Matos MC, Ricardo-Silgado ML, Morales-Álvarez MC, Mantilla-Rivas JO, Pinzón-Cortés JA, et al. Comparison of plasma pigment epithelium-derived factor (PEDF), retinol binding protein 4 (RBP-4), chitinase-3-like protein 1 (YKL-40) and brain-derived neurotrophic factor (BDNF) for the identification of insulin resistance. J Diabetes Complicat. 2017:31:1423-9.

35. Hanson NQ, Freier EF. Nephelometric assay of apolipoprotein A-I with a centrifugal analyzer. Clin Chem. 1986;32:2090-3.

36. Talayero B, Wang L, Furtado J, Carey VJ, Bray GA, Sacks FM. Obesity favors apolipoprotein E- and C-II--containing high density lipoprotein subfractions associated with risk of heart disease. J Lipid Res. 2014;55:2167-77.

37. Zhao Y, Thorngate FE, Weisgraber KH, Williams DL, Parks JS. Apolipoprotein $E$ is the major physiological activator of lecithin-cholesterol acyltransferase (LCAT) on apolipoprotein B lipoproteins. Biochemistry. 2005;44:1013-25.

38. Rye KA, Bright R, Psaltis M, Barter PJ. Regulation of reconstituted high density lipoprotein structure and remodeling by apolipoprotein E. J Lipid Res. 2006:47:1025-36.

39. Nishida HI, Nakanishi T, Yen EA, Arai H, Yen FT, Nishida T. Nature of the enhancement of lecithin-cholesterol acyltransferase reaction by various apolipoproteins. J Biol Chem. 1986;261:12028-35.

40. Millar JS, Lassman ME, Thomas T, Ramakrishnan R, Jumes $P$, et al. Effects of CETP inhibition with anacetrapib on metabolism of VLDL-TG and plasma apolipoproteins C-II, C-III, and E. J Lipid Res. 2017;58:1214-20.

41. Bagdade J, Barter P, Quiroga C, Alaupovic P. Effects of torcetrapib and statin treatment on apoC-III and apoprotein-defined lipoprotein subclasses (from the ILLUMINATE trial). Am J Cardiol. 2017;119:1753-6,

42. Nicholls SJ, Ray KK, Ballantyne CM, Beacham LA, Miller DL, Ruotolo G, et al. Comparative effects of cholesteryl ester transfer protein inhibition, statin or ezetimibe on lipid factors: the ACCENTUATE trial. Atherosclerosis. 2017;261:12-8.

43. Sigal GA, Tavoni TM, Silva BMO, Kalil Filho R, Brandão LG, Maranhão RC. Effects of short-term hypothyroidism on the lipid transfer to high-density lipoprotein and other parameters related to lipoprotein metabolism in patients submitted to thyroidectomy for thyroid cancer. Thyroid. 2019;29: 53-8.

44. Iglesias A, Arranz M, Alvarez JJ, Perales J, Villar J, Herrera E, et al. Cholesteryl ester transfer activity in liver disease and cholestasis, and its relation with fatty acid composition of lipoprotein lipids. Clin Chim Acta. 1996;248:157-74.

45. Huuskonen J, Olkkonen VM, Ehnholm C, Metso J, Julkunen I, Jauhiainen M. Phospholipid transfer is a prerequisite for PLTP-mediated HDL conversion. Biochemistry. 2000;39:16092-8.

46. Rousset X, Shamburek R, Vaisman B, Amar M, Remaley A. Lecithin cholesterol acyltransferase: an anti- or pro-atherogenic factor? Curr Atheroscler Rep. 2011;13:249-56.

47. Sethi A, Sampson M, Warnick R, Muniz N, Vaisman B, Nordestgaard B, et al. High pre- $\beta 1$ HDL concentrations and low lecithin: cholesterol acyltransferase activities are strong positive risk markers for ischemic heart disease and independent of HDL-cholesterol. Clin Chem. 2010;56:1128-37.

48. Sacks FM. The crucial roles of apolipoproteins E and C-III in apoB lipoprotein metabolism in normolipidemia and hypertriglyceridemia. Curr Opin Lipidol. 2015:26:56-63.

49. Morton AM, Koch M, Mendivil CO, Furtado J, Tjønneland A, Overvad K, et al. Apolipoproteins $\mathrm{E}$ and $\mathrm{CIII}$ interact to regulate $\mathrm{HDL}$ metabolism and coronary heart disease risk. JCl Insight. 2018;3:e98045.

50. Morton AM, Furtado JD, Mendivil CO, Sacks FM. Dietary unsaturated fat increases HDL metabolic pathways involving apoE favorable to reverse cholesterol transport. JCl Insight. 2019;4:124620.

\section{Publisher's Note}

Springer Nature remains neutral with regard to jurisdictional claims in published maps and institutional affiliations.

Ready to submit your research? Choose BMC and benefit from:

- fast, convenient online submission

- thorough peer review by experienced researchers in your field

- rapid publication on acceptance

- support for research data, including large and complex data types

- gold Open Access which fosters wider collaboration and increased citations

- maximum visibility for your research: over $100 \mathrm{M}$ website views per year

At BMC, research is always in progress.

Learn more biomedcentral.com/submissions 